\title{
Qualitative Change of the Electronic Properties of a Semiconductor Surface by Chemical Treatment Using Polyelectrolytes
}

\author{
Sabri Akari* \\ Max Planck Institute for Colloids and Interface Science, Rudower Chausse 5, \\ D-12489 Berlin-Adlershof, Germany \\ Thomas W. Matthes, Christof Sommerhalter, J ohannes Boneberg, and \\ Paul Leiderer \\ Fakultät für Physik, Universität Konstanz, D-78434 Konstanz, Germany
}

Received J anuary 16, 1997. In Final Form: May 20, $1997^{\otimes}$

\begin{abstract}
In the present study we demonstrate the ability of changing the el ectronic properties of a semi conductor surface by adsorbing polyel ectrolytes. For this purpose pol yethylenimine (PEI) polymers were adsorbed onto the van der Waals surface of the layered structure semi conductor WSe2. F or comparison the surface properties of the as grown and chemically treated surface were investigated by standard and photoassisted tunneling spectroscopy. In the case of the chemically treated sample a strong change in the I/V characteristics could be observed.
\end{abstract}

\section{Introduction}

The chemical treatment of semiconductor surfaces by different chemical substancestostabilize, improve, or even tune their electronic properties is a field of large inter est. A prominent examplein this areais thesurfacepassivation of silicon by hydrogenation. Concerning the layered structure transition metal dichalcogenides, several experiments are reported in the literature which aim to increasethequantum efficiency of optoel ectronic devices. ${ }^{1,2}$ On thesesemiconductors thechemical treatment by EDTA (ethylenediaminetetracetic acid) or $\mathrm{Nal} / \mathrm{I}_{2}$ results in a passivation of defects which could also be detected as an increase in the photoinduced tunneling current using photoassisted scanning tunneling spectroscopy/microscopy. 3,4 However no qual itative change of the el ectronic surface properties could be observed. In this study we report for the first time on the chemical treatment by adsorbing polyelectrolyte molecules (polyethylenimine, $\mathrm{PEI}$ ) on the layered structure semiconductor $\mathrm{WSe}_{2}$. On thebasis of their large chargedensity in aqueous solution, pureand modified polyethylenimine polymers are widely used in the paper industry as effective drainage and retention aids. In these applications polyethylenimine allows the control of flocculation in stock suspensions. ${ }^{5-7}$ Due to this Iarge charge density they are expected to act indirectly as electron donator with respect to the surface of a p-typesemiconductor. This might result in additional band bending and could increase the open circuit voltage of optoelectronic devices.

\section{Experimental Section}

For our measurements we used a singlecrystal of thetransition metal dichal cogenideWSe ( $p$-type), which was grown by chemical

\footnotetext{
${ }^{\otimes}$ Abstract published in Advance ACS Abstracts, J uly 1, 1997. (1) Hodes, G.; Appl. Phys. Lett. 1989, 54, 2085.

(2) Razzini, G.; Peraldo Bicelli, L. Electrochem. Solar Cells 1981, 128, 2134.

(3) Akari, S.; Lux-Steiner, M. Ch.; Glöckler, K.; Schill, T.; Heikamp, R.; Koslowski, B.; Dransfeld, K.: Ann. Phys. 1993, 2, 141.

(4) Akari, S.; Friemelt, K.; Glöckler, K.; Lux-Steiner, M. Ch.; Bucher, E.; Dransfeld, K. Appl. Phys. 1993, A57, 221.

(5) Horn D.; Linhart, F. Paper chemistry; Roberts, J ., Ed.; Blackie: Glasgow and London, 1991; pp 44-61.

(6) Tankana, H.; Oedberg, L.; Wagberg, L.; Lindström, T. J . Colloid Interface Sci. 134, 1990, 219.

(7) Akari, S.; Schrepp, W.; Horn, D. Langmuir 1996, 12, 857.
}

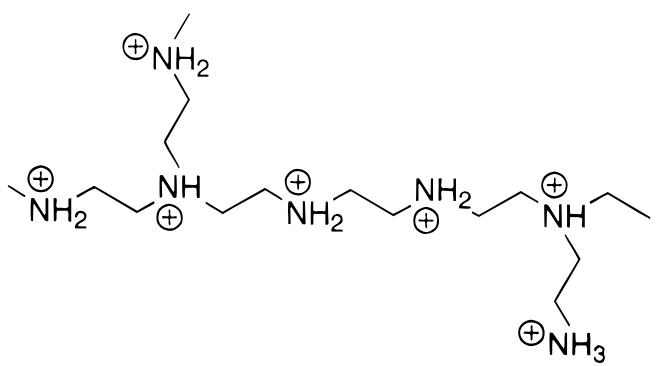

Figure 1. Chemical structure of the PEI molecule, used for the chemical treatment of WSe.

vapor transport. Toallow a direct comparison of the results, the (0001) surface of the same crystal was investigated prior to and after the chemical treatment by standard and photoassisted scanningtunneling spectroscopy. After theinitial measurements on the as grown sample, polyethyleneimine polymers (Figure 1) with a molecular weight of $6200 \mathrm{~g} / \mathrm{mol}$ (BASF AG Ludwigshafen, Germany) werediluted in deionized water ( $50 \mathrm{vol} \%$ ) and dropped onto the crystal surface. After repeatedly rinsing the surface with deionized water, it was dried in a stream of nitrogen.

For theinvestigation of surface properties, weused a standard scanning tunneling microscope which was operated in high vacuum (10-6 Torr). Using mechanically prepared PtI r tips, I/ V characteristics were measured under dark and illuminated conditions (HeNe laser, $633 \mathrm{~nm}, 500 \mathrm{~mW} / \mathrm{cm}^{2}$ ). In order to measure both I/ V characteristics with and without illumination while keeping identical tunneling conditions, we used the following setup: The incident light is modulated by a chopper at a frequency of $500 \mathrm{~Hz}$. During thefirst half of thedark period, the $z$-feedback is set active to adjust the tip-sample separation for a given reference voltage $U_{\text {ref }}$ and reference current $I_{\text {ref. }}$. The $z$-feedback is then switched off and during the second half of the dark period thetunneling current is measured at a fixed tunneling voltage $U_{t}$. The light then turns on and the current at $U_{t}$ is measured under illumination. In the first half of the next dark period the tip-sample distance is readjusted at the reference voltage $U_{\text {ref }}$ and reference current $I_{\text {ref }}$ and so on. By variation of $U_{t}$ the $I / V$ curves under dark and illuminated conditions are thus measured simultaneously for a tip-sampleseparation that is solely determined by $U_{\text {ref }}$ and $I_{\text {ref }}$ under dark conditions. A more detailed description of this setup will be given elsewhere. ${ }^{8}$

(8) Matthes, Th. W.; Sommerhalter, Ch.; Boneberg, J .; Leiderer, P.; Lux-Steiner, M. Ch. To be published. 

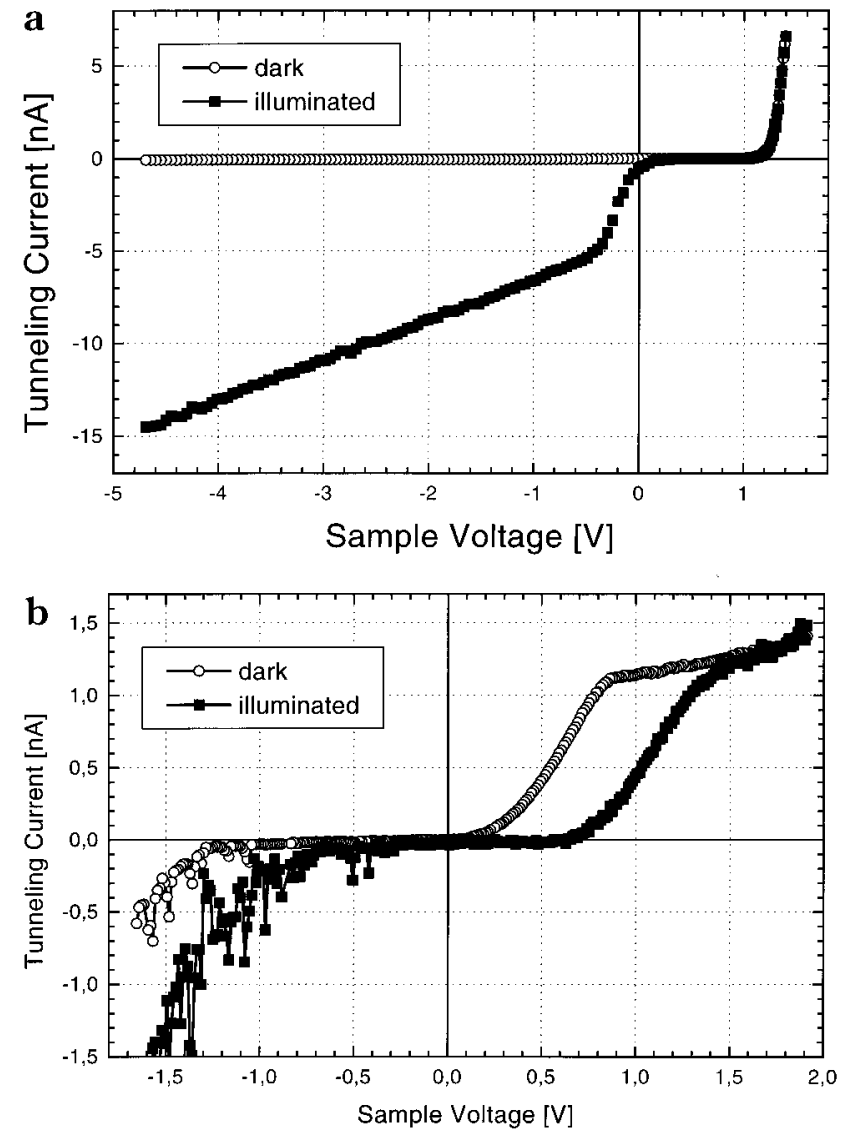

Figure 2. I/ $\mathrm{V}$ characteristics for the as grown (a) and PEI adsorbed (b) WSe $\mathrm{W}_{2}$ single crystal measured by photoassisted tunnel ing spectroscopy. Thetip-sampleseparation is adjusted under dark conditions for an applied reference sample voltage $\mathrm{V}_{\text {ref }}=1.3 \mathrm{~V}$ and reference current $\mathrm{I}_{\text {ref }}=1.2 \mathrm{nA}$. I/ V curves under dark conditions are given by open circles. I/ V curves under illumination (HeNelaser, $633 \mathrm{~nm}, 500 \mathrm{~mW} / \mathrm{cm}^{2}$ ) aregiven by filled squares.

\section{Results}

Prior to the spectroscopic measurements, the surfaces of the as grown and the chemically treated crystal were imaged in the constant current mode. Atomicresolution could be obtained on both surfaces showing the hexagonal structure of the $\mathrm{WSe}_{2}(0001)$ surface. While for the untreated samplevoltages $>+0.8 \mathrm{~V}$ had to beapplied, the treated surface could be atomically resolved at voltages $>+0.2 \mathrm{~V}$. At negative sample voltages no stable imaging conditions could be obtained for both cases. Up to now our attemptstoimagethePEI mol ecules themsel ves failed.

The measured I/ V curves for the as grown and chemically treated surface are shown in parts $a$ and $b$ of Figure 2 , respectively. For the untreated surface the dark I/ V curve exhibits an ideal rectifying behavior. While in the negative voltage range no current could be detected, it increases exponentially in the positivevoltagerange. The I/ V curveunder illumination is identical tothedark curve for voltages between +1.4 and $+0.3 \mathrm{~V}$. At $+0.3 \mathrm{~V}$ we observe the onset of a photoinduced tunneling current, which increases rapidly for decreasing voltageand reaches a regime of smaller linear increase at about $-0.5 \mathrm{~V}$.

The results on the treated surface were completely different (Figure 2). Under dark conditions we observed an exponential increase in the positive voltage range up to $0.8 \mathrm{~V}$ followed by an almost linear regime of much smaller slope for higher voltages. In the negative range an onset of thetunneling current occurs at about $-1.0 \mathrm{~V}$. Weshould notehowever that in thenegativevoltagerange the tunneling current was much less stable compared to positive voltages. A shift by about $0.5 \mathrm{~V}$ to more positive voltages is observed under illumination. At $1.5 \mathrm{~V}$ both curves coal esceand nosignificant photoinduced effect was measured for higher voltages.

\section{Discussion}

In spite of the fact that the measured I/ V curves were completely different after theadsorption of PEI molecules, atomic resolution of the $\mathrm{WSe}_{2}$ lattice could be obtained. Possibly for the applied tunneling conditions the tip penetrates the adsorbed PEI layer until tunneling into the semiconductor can occur. The change of the bias regime at which atomic resolution could be observed reflects the influence of the adsorbed layer onto the semiconductor surface. However, at this stage of experiments and due to the insufficient knowledge about the electronic properties of the PEI molecules an explanation can be given here.

To understand the change of the I/ $\mathrm{V}$ curves due to the adsorption, we will first sketch the basic physical interpretation for the as grown surface. The (0001) van der Waals surface of $\mathrm{WSe}_{2}$ is a typical representative for a semiconductor surface with a very low surface density of states. Duetothelayered structureall bonds of the (0001) surface are saturated and no intrinsic surfacestates have been reported up tonow. ${ }^{9}$ As a consequence, thescanning tunneling microscopy (STM) configuration can be described as a metal-insulator-semiconductor junction with a thin insulating gap. In the proximity of themetallic tip, the band bending at the semiconductor surface is essentially determined by the work function difference between tip and sample and the externally applied tunneling voltage. The band diagrams for different applied potentials are shown in Figure 3. For voltages $<+0.3 \mathrm{~V}$ the bands are bent downward. Light-generated minority charge carriers are partially driven to the semiconductor surface, where they can be detected as an additional light-generated (negative) tunneling current or they are lost by backdiffusion and recombination in the bulk. Theregime of quasi-saturation of thephotoinduced tunneling current (below $-0.5 \mathrm{~V}$ ) is reached, when the amount of minorities that are lost by backdiffusion becomes negligible due to the extended space charge region. In thissituation all minority chargecarriers which are driven to the space charge region will contribute to the photoinduced tunneling current. At voltages $>+0.3 \mathrm{~V}$ the bands are bent upward. Now the hol es are driven to the surface, while the electrons are driven into the bulk. Thefact that nopositive photoinduced current is detected can be explained by the negligible photoinduced change of the majority charge carrier concentration for moderate or high doping levels. A more detailed discussion as well as a one-dimensional model calculation will be given in ref $10^{10}$ for the case of the isostructural $W_{2}$.

For the case of the PEI-adsorbed WSe $\mathrm{W}_{2}$ surface, we can give only a preliminary interpretation.

From our experiments we can conclude that the main effect of the adsorbed layer is a transfer of electrons into the p-type semiconductor. Sincein the presence of water molecules the polyethylenimin (basicsubstance) is a proton acceptor, the excess of negatively charged $\mathrm{OH}$ groups might act as electron donator. However one could also think of a direct el ectron transfer which solely involves thePEI molecules themselves. Up tonow wedonot know whether a water layer is still present under the high vacuum conditions, that we used for the STM measurements.

(9) Mayer, T.; Klein, A.; Lang, O.; J ägermann, W. Surf. Sci. 1991 269/270, 909 and references herein.

(10) Sommerhalter, Ch.; Matthes, Th. W.; Boneberg, J .; Leiderer, P.; Lux-Steiner, M. Ch. Submitted to J . Vac. Sci. Technol. B. 

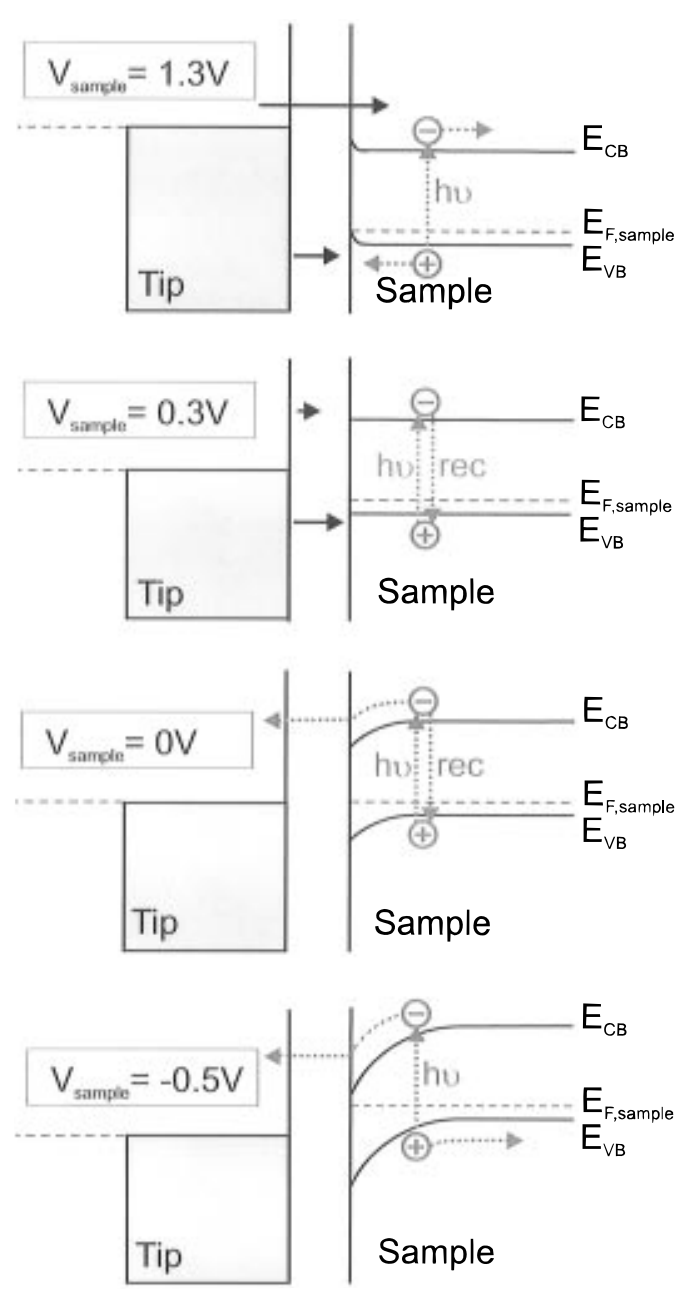

Figure 3. Band diagrams for the tip/vacuum/p-WSe system $^{2}$ prior to the PEI treatment. The charge carrier transport channels are given by arrows (black solid arrows for transport that occurs under dark conditions as well as gray dotted arrows for transport that involves light generated charge carriers). Under illumination and in case of upward band bending (upper diagram) electrons are driven to the bulk and holes to the surface; in thecase of downward band bending (lower diagrams) el ectrons are driven to the surface and holes to the bulk. In both cases a photoinduced tunneling current is resulting.

In both cases an additional downward band bending at the semi conductor surface is expected. Extrinsic surface states can becreated at thePEI -semiconductor interface, which act as recombination centers and effectively pin thesemiconductor Fermi level. As a consequencethel/ V characteristic should be comparable to that of a conventional semiconductor surface with Fermi level pinning due to intrinsic surface states (for example Si(111)-(7 x 7) ${ }^{11}$ ). Under these conditions, the applied voltage essentially drops within the tunneling gap. The band diagram for zero applied voltage is shown in Figure 4. By variation of the tunneling vol tage, the tunneling current probes theavailabledensity of states, i.e., theempty states in thesemiconductor's conduction band at positivesample voltages and the density of filled states in the valence band at negative voltages. Therefore the measured low current regimecorresponds tothesemiconductor bandgap in this model.

Under illumination an additional surface photovoltage shifts the I-V curveto more positive potential. The band bending is reduced due to the photogenerated minority charge carriers which are subsequently trapped and recombine via surface states. The shift of the I-V curve

(11) Kuk, Y.; Becker, R. S.; Silverman, P.J .; Kochanski, G. P.J . Vac. Sci. Technol. 1991, B9, 545.
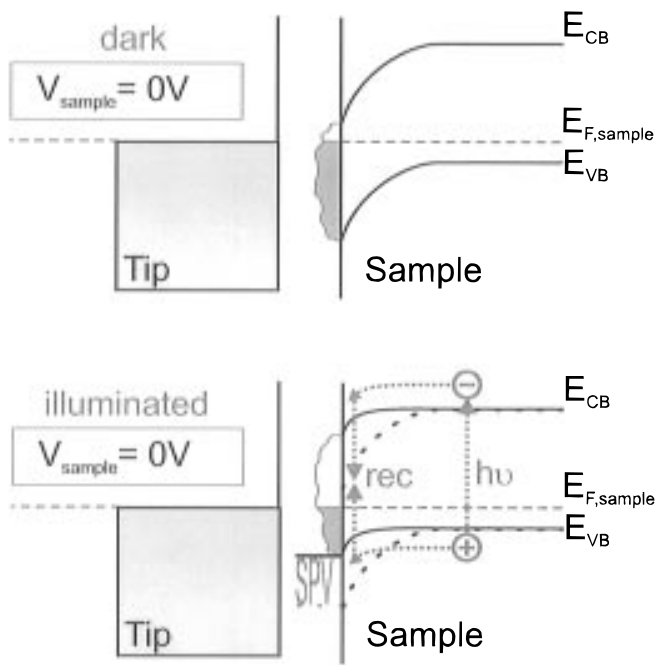

Figure4. Band diagramfor thecase of thePEI-treated surface. Thesemiconductors Fermi level is effectively pinned by surface states. Under illumination the band bending is reduced due to the photogenerated minority charge carriers which are subsequently trapped and recombine via surface states due to the PEI treatment. SPV corresponds to surface photo vol tage and rec to recombination.

under illumination gives a lower limit for the band bending due to the adsorption of PEI.

The behavior of the dark current in the voltage range between 0.9 and $1.5 \mathrm{~V}$ might be a consequence of the minority injection from the tip. ${ }^{12}$ If the current density of el ectrons, which are injected into the conduction band of the semiconductor by the tunneling current, increases toward higher voltages, some of these charge carriers can return tothesurface. Therethey will betrapped by surface states and reduce the band bending in the same way as electrons, which are created by photoexcitation.

If our simple model is valid, the shift of the I-V curves under illumination should depend on the light intensity and the coal escence of dark and illuminated curve at high positive voltages should depend on the magnitude of the tunneling current. However, at this stage of our investigations we cannot exclude that the observed behavior might also be associated with tunneling via the electronic states of thePEI molecules. Further detailed experiments are necessary to clarify the responsible process.

\section{Conclusions}

In summary we have demonstrated that the adsorption of PEI molecules ontothevan der Waals surface of a p-type $\mathrm{WSe}_{2}$ single crystal essentially changes the electronic surface properties. From the results of our photoassisted scanning tunneling spectroscopy investigations, we can conclude that the adsorption of PEI induces a strong downward band bending at the semiconductor surface, which might be of interest for theoptimization of electronic and optoel ectronic devices. Further experiments will be necessary to clarify the underlying electronic processes and to investigate the technological potential of the observed effects.

Acknowledgment. We thank the BASF AG (Ludwigshafen) for providing us with the polyethylenimine samples and Professor Möhwald for useful discussions and support.

\section{LA970048Z}

(12) Cahill, D. G.; Feenstra, R. M. J . Vac. Sci. Technol. 1993, A11, 